\title{
A sharper threshold for bootstrap percolation in two dimensions
}

\author{
Janko Gravner • Alexander E. Holroyd • \\ Robert Morris
}

Received: 20 February 2010 / Revised: 26 October 2010 / Published online: 31 December 2010 C The Author(s) 2010. This article is published with open access at Springerlink.com

\begin{abstract}
Two-dimensional bootstrap percolation is a cellular automaton in which sites become 'infected' by contact with two or more already infected nearest neighbours. We consider these dynamics, which can be interpreted as a monotone version of the Ising model, on an $n \times n$ square, with sites initially infected independently with probability $p$. The critical probability $p_{c}$ is the smallest $p$ for which the probability that the entire square is eventually infected exceeds $1 / 2$. Holroyd determined the sharp first-order approximation: $p_{c} \sim \pi^{2} /(18 \log n)$ as $n \rightarrow \infty$. Here we sharpen this result, proving that the second term in the expansion is $-(\log n)^{-3 / 2+o(1)}$, and moreover determining it up to a poly $(\log \log n)$-factor. The exponent $-3 / 2$ corrects numerical predictions from the physics literature.
\end{abstract}

Supported by NSF grant DMS 0204376 and the Republic of Slovenia Ministry of Science program P1-285 (JG); NSERC and Microsoft Research (AEH); a JSPS Fellowship and a Research Fellowship from Murray Edwards College, Cambridge (RM).

\section{J. Gravner}

Mathematics Department, University of California,

Davis, CA 95616, USA

e-mail: gravner@math.ucdavis.edu

A. E. Holroyd

Microsoft Research, 1 Microsoft Way,

Redmond, WA 98052, USA

\section{A. E. Holroyd}

University of British Columbia, 121-1984 Mathematics Road,

Vancouver, BC V6T 1Z2, Canada

e-mail: holroyd@math.ubc.ca

R. Morris $(\varangle)$

IMPA, Estrada Dona Castorina 110, Jardim Botânico,

Rio de Janeiro, RJ, Brazil

e-mail:rob@impa.br 
Mathematics Subject Classification (2000) $\quad 60 \mathrm{C} 05 \cdot 60 \mathrm{~K} 35 \cdot 82 \mathrm{~B} 20$

\section{Introduction}

Bootstrap percolation is a cellular automaton in which, given a (typically random) initial set of 'infected' vertices in a graph $G$, new vertices are infected at each time step if they have at least $r$ infected neighbours. In this paper we shall study two-neighbour bootstrap percolation on the square grid $[n]^{2}$. We shall determine the second term of the critical threshold for percolation up to a poly $(\log \log n)$-factor, and hence confirm a conjecture of Gravner and Holroyd [29].

We begin by defining the bootstrap process, which was introduced by Chalupa et al. [19] in 1979. Let $G$ be a graph with vertex set $V(G)$, and for each vertex $v \in V(G)$, let $N(v)$ denote the set of neighbours of $v$ in $G$. Now, given an integer $r \in \mathbb{N}$, and a set of initially infected vertices $A \subset V(G)$, define $A_{t}$ recursively as follows: set $A_{0}=A$, and

$$
A_{t+1}=A_{t} \cup\left\{v \in V(G):\left|N(v) \cap A_{t}\right| \geq r\right\}
$$

for each integer $t \geq 0$. We say that the vertices of $A_{t}$ have been infected by time $t$. Let $[A]=\bigcup_{t} A_{t}$ denote the closure of $A$ under the $r$-neighbour bootstrap process, and say that the set $A$ percolates if the entire vertex set is eventually infected, i.e., if $[A]=V(G)$.

We shall be interested in the case in which $A$ is a random subset of $V(G)$. More precisely, let us choose the elements of $A$ independently at random, each with probability $p$, and denote by $\mathbb{P}_{p}$ the corresponding probability measure. Throughout the paper, $A$ will be assumed to be a random subset selected according to this distribution, unless otherwise stated. It is clear that the probability of percolation is increasing in $p$, and so we define the critical probability, $p_{c}(G, r)$ as follows:

$$
p_{c}(G, r):=\inf \left\{p: \mathbb{P}_{p}(A \text { percolates in the } r \text {-neighbour process on } G) \geq 1 / 2\right\}
$$

Our aim is to give sharp bounds on $p_{c}(G, r)$.

Bootstrap percolation has been studied extensively by mathematicians $[2,6,16,31$, $40]$, as well as by physicists $[1,11,30]$ and sociologists $[26,41]$, amongst others. The bootstrap process was originally introduced in the context of disordered magnetic systems, and may be thought of as a monotone version of the Glauber dynamics of the Ising model. Indeed, if spins are allowed to flip in only one direction (from - to + , say), and if they flip only if they have at least $r$ neighbours in state + , then one immediately obtains the cellular automaton described above. We refer the interested reader to the applications of bootstrap percolation in [24,38], and the closely related models studied in $[18,20,21,27,36,39]$.

We focus on the graph $G=[n]^{d}$ with vertex set $\{1, \ldots, n\}^{d}$, and with an edge between vertices $u$ and $v$ if and only if $\|u-v\|_{1}=1$. Aizenman and Lebowitz [2] determined the asymptotic behaviour of $p_{c}\left([n]^{d}, 2\right)$ up to multiplicative constants, and Cerf and Cirillo [16] (in the crucial case $d=r=3$ ) and Cerf and Manzo [17] proved 
the corresponding result for all $d \geq r \geq 2$. The first sharp threshold for bootstrap percolation was proved by Holroyd [31], who showed that

$$
p_{c}\left([n]^{2}, 2\right)=\frac{\pi^{2}}{18 \log n}+o\left(\frac{1}{\log n}\right)
$$

This was the first result of its type, and has prompted a flurry of generalizations. Sharp thresholds have since been determined for $p_{c}\left([n]^{d}, r\right)$ for all fixed $d$ and $r$ [6,8], for more general update rules in two dimensions [22], and in high dimensions (i.e., $d=d(n) \rightarrow \infty$ sufficiently fast) for the majority rule, i.e., $r=d$ [5], and in the case $r=2$ [7]. Some of the techniques from these papers have been used to prove results about the Glauber dynamics of the Ising model [24,38]. The bootstrap process has also been studied on infinite trees [9,13,23], on 'locally tree-like' graphs [5], on the random regular graph [10,34], and on the Erdős-Rényi random graph $G_{n, p}$ [35].

In this paper we shall study the two-neighbour bootstrap process on the graph $G=[n]^{2}$ in more detail. One of the most striking facts about the result (1) stated above is that it contradicted estimates of $\lim _{n \rightarrow \infty} p_{c} \log n$ given by simulations-in fact, such estimates were out by a factor of more than two. (See, for example, [28] or [30] for a discussion of the reasons behind these discrepancies.) Gravner and Holroyd [28] gave a rigorous (partial) explanation for this phenomenon, by giving the following improvement of (1):

$$
p_{c}\left([n]^{2}, 2\right) \leq \frac{\pi^{2}}{18 \log n}-\frac{c}{(\log n)^{3 / 2}},
$$

where $c>0$ is a small constant. In [29], the same authors proved an almost matching lower bound for a simpler model (called 'local' bootstrap percolation), and conjectured that the upper bound is essentially sharp for the usual bootstrap process.

Conjecture 1 (Gravner and Holroyd [29]) For every $\varepsilon>0$, if $n$ is sufficiently large then

$$
p_{c}\left([n]^{2}, 2\right) \geq \frac{\pi^{2}}{18 \log n}-\frac{1}{(\log n)^{3 / 2-\varepsilon}} .
$$

In this paper we shall prove Conjecture 1 in a slightly stronger form. To be precise, we shall prove the lower bound in the following theorem; the upper bound was proved in [28].

Theorem 1 There exist constants $C>0$ and $c>0$ such that

$$
\frac{\pi^{2}}{18 \log n}-\frac{C(\log \log n)^{3}}{(\log n)^{3 / 2}} \leq p_{c}\left([n]^{2}, 2\right) \leq \frac{\pi^{2}}{18 \log n}-\frac{c}{(\log n)^{3 / 2}}
$$

for every sufficiently large $n \in \mathbb{N}$. 
Our result again corrects numerical predictions, this time for the power of $\log n$ in the second term. Specifically, in work reported in [1], Stauffer interpolated between simulations and the rigorous result (1) to obtain the prediction

$$
p_{c}\left([n]^{2}, 2\right) \approx \frac{\pi^{2}}{18 \log n}-\frac{0.45}{(\log n)^{1.2}},
$$

which is inconsistent with the lower bound in Theorem 1 in the limit $n \rightarrow \infty$. Since $3 / 2>1.2$, the true asymptotic approach to the first approximation $\pi^{2} /(18 \log n)$ is faster than in the above numerical prediction. Furthermore, in Sect. 5 we shall discuss how the proof of Theorem 1 can be adapted to a variant of bootstrap percolation called the Froböse model. In this case, the resulting power $3 / 2$ of $\log n$ corrects the prediction 1.388 made in [30] on the basis of computer calculations for the local Froböse model.

The proof of Theorem 1 will use many of the tools and techniques of [31], together with some of the ideas of [29], and some new ideas. In particular, we shall bound the probability of percolation by the expected number of 'good' and 'satisfied' hierarchies (see Lemma 7, below). We will define a hierarchy as in [31] (see Sect. 3), except that our hierarchies will be much finer, each step being of order $1 / \sqrt{p}$, instead of $1 / p$. This means that we will have far too many hierarchies; however, almost all of these have many 'large' seeds, and we shall show that these contribute a negligible amount to the sum. In order to do so, we shall need a better bound on the probability that a seed is internally spanned than the straightforward bound that sufficed in [31]. Fortunately, the bound we need follows easily from the simple (folklore) fact that a spanning set for a rectangle $R$ must contain no fewer than $\phi(R) / 2$ elements, where $\phi(R)$ denotes the semi-perimeter of $R$ (see Lemmas 2 and 3 ). Surprisingly, it appears that our proof does not extend directly to the "modified" bootstrap percolation model; it is the analogous bound for seeds that is missing in this case (see Sect. 5 for more information).

We finish this section by making a few definitions which we shall use throughout the proof. First, we say a set $S$ is spanned by the set $A$ if $S \subset[A]$, and that $S$ is internally spanned by $A$ if $S \subset[A \cap S]$. An event is simply a collection $\mathcal{A}$ of subsets of $[n]^{2}$; we say $\mathcal{A}$ holds if $A \in \mathcal{A}$. In order to keep our formulae relatively compact, we shall sometimes write $I(S)$ for the event that $S$ is internally spanned by $A$.

Next, define two functions, $\beta$ and $g$, by

$$
\beta(u):=\frac{u+\sqrt{u(4-3 u)}}{2} \text { and } g(z):=-\log \left(\beta\left(1-e^{-z}\right)\right)
$$

We remark that $\beta$ is increasing on $[0,1]$, and so $g$ is decreasing on $(0, \infty)$, and that $g(z) \leq 2 e^{-z}$ when $z$ is large (see Proposition 3 of [6]). Note that $\beta(u) \sim \sqrt{u}$ as $u \rightarrow 0$, and so $g(z) \sim-\log \sqrt{z}$ as $z \rightarrow 0$, where $g(z) \sim h(z)$ means that $g(z) / h(z) \rightarrow 1$.

A rectangle is a set of the form

$$
R=[(a, b),(c, d)]:=\{(x, y): a \leq x \leq c, b \leq y \leq d\} \subset \mathbb{Z}^{2}
$$

where $a, b, c, d \in \mathbb{Z}$. The dimensions of $R$ are $\operatorname{dim}(R)=(c-a+1, d-b+1)$, the long and short side-lengths of $R$ are respectively $\operatorname{sh}(R)=\min \{c-a+1, d-b+1\}$ 
and $\lg (R)=\max \{c-a+1, d-b+1\}$, and the semi-perimeter of $R$ is $\phi(R)=$ $\operatorname{sh}(R)+\lg (R)$.

We say that a rectangle $R=[(a, b),(c, d)]$ is crossed from left-to-right by $A \subset R$ if

$$
R \subset\left[A \cup\left\{(x, y) \in \mathbb{Z}^{2}: x \leq a-1\right\}\right],
$$

i.e., if $R$ is spanned by $A$ together with the set of all sites to the left of $R$. Note that this is equivalent to there being no 'double gap' (i.e., no adjacent 'empty' pair of columns) in $R$, and the final column being occupied. (Here 'empty' means 'contains no element of $A$ ' and 'occupied' means 'not empty'.)

For each $p \in(0,1)$, let $q=-\log (1-p)$, so that $p \sim q$ as $p \rightarrow 0$. To motivate this definition (and the definition of $g(z)$, above), note (from Lemma 8 of [31]) that for any rectangle $R$ with dimensions $(a, b)$, then

$$
\mathbb{P}_{p}(A \text { crosses } R \text { from left-to-right }) \leq e^{-a g(b q)} .
$$

We shall use the notation $f(\mathbf{x})=O(h(\mathbf{x}))$ throughout to mean that there exists an absolute constant $C>0$, independent of all other variables (unless otherwise stated), such that $f(\mathbf{x}) \leq C h(\mathbf{x})$ for all $\mathbf{x}=\left(x_{1}, \ldots, x_{k}\right)$. If the constant $C$ depends on some other parameter $y$, then we shall write $f(\mathbf{x})=O_{y}(h(\mathbf{x}))$.

We shall write $\mathbb{R}_{+}$for the positive reals, and if $\mathbf{a}, \mathbf{b} \in \mathbb{R}^{2}$, then we shall write $\mathbf{a} \leq \mathbf{b}$ if $a_{1} \leq b_{1}$ and $a_{2} \leq b_{2}$. Thus a path in $\mathbb{R}^{2}$ is 'increasing' if it is increasing in both coordinates. Finally, if $G$ is a directed graph, then $\vec{N}_{G}(v)$ will denote the set of out-neighbours of a vertex $v$ in $G$.

The rest of the paper is organised as follows. In Sect. 2 we give an upper bound on the probability that a sufficiently small rectangle (a seed) is internally spanned. In Sect. 3 we recall from [31] the notion of a hierarchy, which is fundamental to the proof of Theorem 1, together with some important lemmas from [29,31]. In Sect. 4 we prove Theorem 1, and in Sect. 5 we mention some open questions.

\section{A lemma on seeds}

In this section we shall prove the following lemma, which bounds the probability that a small rectangle is internally spanned. Recall that $q=-\log (1-p)$.

Lemma 2 There exists $\delta>0$ such that, for any $p>0$, and any rectangle $R$ with $\operatorname{dim}(R)=(a, b)$, where $a \leq b$ and $a p \leq \delta$ then

$$
\mathbb{P}_{p}([A \cap R]=R) \leq 3^{\phi(R)} \exp (-\phi(R) g(a q)) .
$$

We begin by recalling a lovely and well-known exercise for high school students (see [14] or [42], for example). Lemma 2 follows from it almost immediately.

Lemma 3 If $R$ is a rectangle, and A internally spans $R$, then $|A \cap R| \geq \phi(R) / 2$.

We also make a simple observation. 
Observation 4 If $z>0$ is sufficiently small then

$$
\log (1 / \sqrt{z})-\sqrt{z} \leq g(z) \leq \log (1 / \sqrt{z})+z
$$

Proof We use the estimates $z-z^{2} \leq 1-e^{-z} \leq z$, and $\sqrt{u} \leq \beta(u) \leq \sqrt{u}+u$, which are valid for small $z$ and $u$. It follows that

$$
g(z) \geq-\log \beta(z) \geq-\log (\sqrt{z}+z)=-\log \sqrt{z}-\log (1+\sqrt{z}) \geq-\log \sqrt{z}-\sqrt{z}
$$

The proof of the upper bound is similar.

We can now easily deduce Lemma 2.

Proof of Lemma 2. Let $m=|A \cap R|$. By Lemma 3, if $A$ internally spans $R$ then $m \geq(a+b) / 2$. There are at most $\left(\begin{array}{l}a b \\ m\end{array}\right)$ ways to choose the set $A \cap R$, given $m$, and each occurs with probability at most $p^{m}$. Thus, by the union bound,

$$
\mathbb{P}_{p}([A \cap R]=R) \leq \sum_{m \geq(a+b) / 2}\left(\begin{array}{c}
a b \\
m
\end{array}\right) p^{m} \leq(6 a q)^{(a+b) / 2}
$$

The final inequality follows since $\left(\begin{array}{c}a b \\ m\end{array}\right) p^{m} \leq\left(\frac{e a b p}{m}\right)^{m} \leq(6 a q)^{m} / 2$ if $\delta>0$ is sufficiently small, and since $6 a q \leq 12 \delta<1 / 2$. In the second inequality we used $p \sim q$ and $m \geq b / 2$.

But $\log (1 / \sqrt{a q}) \geq g(a q)-a q$, by Observation 4, so

$$
(a q)^{(a+b) / 2} \leq \exp (-(a+b) g(a q)+(a+b) a q) .
$$

The result now follows, since $a q \leq 2 \delta$, and $\sqrt{6} e^{2 \delta}<3$ if $\delta$ is sufficiently small.

\section{Hierarchies}

In this section we shall recall some important definitions and lemmas from [29] and [31]; for the proofs, we refer the reader to those papers. In particular, we define a hierarchy as in Sect. 9 of [31].

Definition A hierarchy $\mathcal{H}$ for a rectangle $R \subset[n]^{2}$ is an oriented rooted tree $G_{\mathcal{H}}$, with all edges oriented away from the root ('downwards'), together with a collection of rectangles $\left(R_{u} \subset[n]^{2}: u \in V\left(G_{\mathcal{H}}\right)\right)$, one for each vertex of $G_{\mathcal{H}}$, satisfying the following criteria.

(a) The root of $G_{\mathcal{H}}$ corresponds to $R$.

(b) Each vertex has at most 2 neighbours below it.

(c) If $v \in \vec{N}_{G_{\mathcal{H}}}(u)$ then $R_{u} \supset R_{v}$.

(d) If $\vec{N}_{G_{\mathcal{H}}}(u)=\{v, w\}$ then $\left[R_{v} \cup R_{w}\right]=R_{u}$. 
A vertex $u$ with $\vec{N}_{G_{\mathcal{H}}}(u)=\emptyset$ is called a seed. Given two rectangles $S \subset R$, we write $D(S, R)$ for the event (depending on the set $(A \cap R) \backslash S)$ that

$$
R=[(A \cup S) \cap R],
$$

i.e., the event that $R$ is internally spanned by $A \cup S$.

An event $\mathcal{A}$ is increasing if $A \in \mathcal{A}$ and $A \subset A^{\prime}$ implies that $A^{\prime} \in \mathcal{A}$. Two increasing events $\mathcal{B}$ and $\mathcal{C}$ are said to occur disjointly if there exist disjoint sets $B \subset A$ and $C \subset A$ with $B \in \mathcal{B}$ and $C \in \mathcal{C}$. We write $\mathcal{B} \circ \mathcal{C}$ for the collection of such sets $A$, i.e., the event that $\mathcal{B}$ and $\mathcal{C}$ occur disjointly. We say a hierarchy occurs (or is satisfied by the set $A$ ) if the following events all occur disjointly.

(e) For each seed $u: R_{u}$ is internally spanned by $A$.

(f) For each pair $(u, v)$ satisfying $\vec{N}_{G_{\mathcal{H}}}(u)=\{v\}: D\left(R_{v}, R_{u}\right)$ occurs.

Given two rectangles $S \subset R$, with dimensions $\left(a_{1}, a_{2}\right)$ and $\left(b_{1}, b_{2}\right)$ respectively, define

$$
d_{j}(S, R):=\frac{b_{j}-a_{j}}{b_{j}}
$$

for $j=1,2$, and let $d(S, R)=\max \left\{d_{1}(S, R), d_{2}(S, R)\right\}$.

The following definition is slightly different to that in [31], and is motivated by the method of [29] (see also Lemma 9 below). This definition is necessary because in order to prove a sharper result, we need to take a finer hierarchy. In our application we shall take $T=\sqrt{q}$ and $Z=\log ^{3}(1 / q) / \sqrt{q}$.

Definition A hierarchy is $\operatorname{good}$ for $(T, Z) \in \mathbb{R}_{+}^{2}$ if is satisfies the following.

(g) If $\vec{N}_{G_{\mathcal{H}}}(u)=\{v\}$ and $\left|\vec{N}_{G_{\mathcal{H}}}(v)\right|=1$ then $T \leq d\left(R_{v}, R_{u}\right) \leq 2 T$.

(h) If $\vec{N}_{G_{\mathcal{H}}}(u)=\{v\}$ and $\left|\vec{N}_{G_{\mathcal{H}}}(v)\right| \neq 1$ then $d\left(R_{v}, R_{u}\right) \leq 2 T$.

(i) If $\left|\vec{N}_{G_{\mathcal{H}}}(u)\right|=2$ and $v \in \vec{N}_{G_{\mathcal{H}}}(u)$, then $d\left(R_{v}, R_{u}\right) \geq T$.

(j) $u$ is a seed if, and only if, $\operatorname{sh}\left(R_{u}\right) \leq Z$.

Before continuing, we make a simple observation about the height, $h(\mathcal{H})$ of a hierarchy $\mathcal{H}$, by which we mean the maximum distance in $G_{\mathcal{H}}$ of a leaf from the root.

Lemma 5 Let $R$ be a rectangle, let $Z>1>T>0$, and let $\mathcal{H}$ be a hierarchy for $R$ which is good for $(T, Z)$. Then

$$
h(\mathcal{H}) \leq \frac{8}{T} \log \left(\frac{\phi(R)}{Z}\right)+1 .
$$

Proof Consider a path $P$ of length $h(\mathcal{H})$ from the root to a leaf $u$. Let $w$ be the parent (i.e., the neighbour) of $u$ in $G_{\mathcal{H}}$, and note that $\operatorname{sh}\left(R_{w}\right)>Z$. Note also that in every two steps backwards along $P$, at least one of the dimensions of the corresponding rectangle increases by a factor of at least $1+T$. Hence one of the dimensions goes up by this factor at least $(h(\mathcal{H})-1) / 4$ times (on the path from $w$ to the root), and so

$$
Z(1+T)^{(h(\mathcal{H})-1) / 4} \leq \phi(R) .
$$


The result follows by rearranging and using the inequality $\log (1+T) \geq T / 2$, which is valid for all $T \in(0,1)$.

The following key lemma about hierarchies was proved in [31]. Although our definition of hierarchy is slightly different, the proof in our case is almost identical.

Lemma 6 (Proposition 32 of [31]) Let $Z>1>T>0$, let $R$ be a rectangle, and suppose A internally spans $R$. Then there exists a hierarchy $\mathcal{H}$ for $R$, which is good for $(T, Z)$, and which is satisfied by $A$.

Sketch of proof. We use induction on $\phi(R)$; if $\phi(R) \leq Z$ then the result is trivial. Now, assume $\phi(R)>Z$ and apply Proposition 30 of [31] (see also [3] or [4]) repeatedly, each time choosing the rectangle $S$ which minimizes $d(S, R)$. We stop when we obtain a rectangle $S$ such that either $\phi(S) \leq Z$, or $d(S, R) \geq T$.

There are three cases. If $\phi(S) \leq Z$ and $\bar{d}(S, R) \leq 2 T$, then $\mathcal{H}$ has two vertices. If $\phi(S)>Z$ and $d(S, R) \leq 2 T$, the root of $\mathcal{H}$ has degree one, and the rest of $\mathcal{H}$ can be found by applying the induction hypothesis to $S$.

So assume that $d(S, R)>2 T$, and consider the last application of Proposition 30 of [31]. We deduce that there exist rectangles $S^{\prime}$ and $U$ with $\left[S \cup S^{\prime}\right]=U$, with $d(U, R) \leq T, \phi(U)>Z$ and $d\left(S^{\prime}, R\right)>2 T$, and such that $S$ and $S^{\prime}$ are disjointly internally spanned by $A$. But $d(S, U) \geq d(S, R)-d(U, R) \geq T$, and similarly for $S^{\prime}$. Thus, applying the induction hypothesis to $S$ and $S^{\prime}$, we obtain a hierarchy $\mathcal{H}$ as required.

Finally, recall the following fundamental lemma of van den Berg and Kesten [12].

The van den Berg-Kesten Lemma. Let $\mathcal{A}$ and $\mathcal{B}$ be any two increasing events, and let $p \in(0,1)$. Then

$$
\mathbb{P}_{p}(\mathcal{A} \circ \mathcal{B}) \leq \mathbb{P}_{p}(\mathcal{A}) \mathbb{P}_{p}(\mathcal{B})
$$

We can now easily deduce, as in Sect. 10 of [31], our basic bound on the probability of percolation. Given a rectangle $R$ and a pair $(T, Z) \in \mathbb{R}^{2}$, we write $\mathcal{H}(R, T, Z)$ for the collection of hierarchies for $R$ which are good for $(T, Z)$.

Recall that $\mathbb{P}_{p}(I(R))$ and $\mathbb{P}_{p}(D(S, R))$ denote the probabilities in $\mathbb{P}_{p}$ of the events " $R$ is internally spanned by $A$ " and " $R$ is internally spanned by $A \cup S$ " respectively.

Lemma 7 Let $R$ be a rectangle in $[n]^{2}$, let $Z>1>T>0$, and let $p>0$. Then

$$
\mathbb{P}_{p}([A \cap R]=R) \leq \sum_{\mathcal{H} \in \mathcal{H}(R, T, Z)}\left(\prod_{\vec{N}_{G}(u)=\{v\}} \mathbb{P}_{p}\left(D\left(R_{v}, R_{u}\right)\right)\right)\left(\prod_{\text {seeds } u} \mathbb{P}_{p}\left(I\left(R_{u}\right)\right)\right)
$$

(Above and in subsequent usage, the first product is over all pairs of vertices $(u, v)$ of $\mathcal{H}$ that satisfy the given condition $\vec{N}_{G_{\mathcal{H}}}(u)=\{v\}$, and the second product is over all seeds $u$ of $\mathcal{H}$.) 
Proof of Lemma 7. By Lemma 6, if $A$ internally spans $R$ then there exists a hierarchy in $\mathcal{H}(R, T, Z)$ which is satisfied by $A$. Hence the probability that $A$ internally spans $R$ is bounded above by the expected number of such hierarchies. Since the events $I\left(R_{u}\right)$ and $D\left(R_{v}, R_{u}\right)$ are all monotone, and all occur disjointly (see $(e)$ and $(f)$ above), the result follows by the van den Berg-Kesten Lemma.

We recall the following lemma of Aizenman and Lebowitz [2], which is a standard tool for proving lower bounds on $p_{c}\left([n]^{d}, 2\right)$.

Lemma 8 Suppose A internally spans $[n]^{2}$. Then, for all $1 \leq L \leq n$, there exists a rectangle $R$, internally spanned by $A$, with

$$
L \leq \lg (R) \leq 2 L
$$

We recall also the following bound on $\mathbb{P}_{p}\left(D\left(R, R^{\prime}\right)\right)$ from [29].

Lemma 9 (Lemma 5 of [29]) Let $R \subset R^{\prime}$ be rectangles of dimensions $(a, b)$ and $(a+s, b+t)$ respectively, and let $p>0$. Then

$\mathbb{P}_{p}\left(D\left(R, R^{\prime}\right)\right) \leq \exp \left(-s g(b q)-t g(a q)+2(g(b q)+g(a q))+(q s t) e^{2 g(b q)+2 g(a q)}\right)$.

The following observation follows exactly as in Lemma 10 of [29].

Observation 10 (Lemma 10 of [29]) Let $B>0$ be sufficiently large, and let a $\in \mathbb{N}$ and $q>0$ satisfy $a \leq B / q$. Then

$$
e^{2 g(a q)} \leq \frac{4 B}{a q}
$$

Proof Let $z>0$ and $u=1-e^{-z}$, and recall that $e^{-g(z)}=\beta(u)$. Recall also that $\beta(u) \sim \sqrt{z}$ when $z \rightarrow 0$ and that $\beta(u) \rightarrow 1$ as $z \rightarrow \infty$. Thus, since $B>0$ is sufficiently large, it follows that

$$
\beta(u) \geq \frac{1}{2} \sqrt{\frac{z}{B}}
$$

for every $z \leq B$, as required.

We shall need a couple more definitions in order to rewrite Lemmas 7 and 9 in a more useful form. Given $\mathbf{a}, \mathbf{b} \in \mathbb{R}_{+}^{2}$ with $\mathbf{a} \leq \mathbf{b}$, let

$$
W_{g}(\mathbf{a}, \mathbf{b}):=\inf _{\gamma: \mathbf{a} \rightarrow \mathbf{b}} \int_{\gamma}(g(y) d x+g(x) d y),
$$

where the infimum is taken over all piecewise linear, increasing paths from $\mathbf{a}$ to $\mathbf{b}$ in $\mathbb{R}^{2}$ (see Sect. 6 of [31]). Now, for any two rectangles $R \subset R^{\prime}$, and given $p>0$, define 


$$
U\left(R, R^{\prime}\right)=W_{g}\left(q \operatorname{dim}(R), q \operatorname{dim}\left(R^{\prime}\right)\right) .
$$

The following observation is immediate from the definition.

Observation 11 (Proposition 13 of [31]) Let $R \subset R^{\prime}$ be rectangles of dimensions $(a, b)$ and $(a+s, b+t)$ respectively, and let $p>0$. Then

$$
s g(b q)+\operatorname{tg}(a q) \geq \frac{1}{q} U\left(R, R^{\prime}\right)
$$

Let $N(\mathcal{H})$ denote the number of vertices in a hierarchy $\mathcal{H}$, and $M(\mathcal{H})$ denote the number of vertices of $\mathcal{H}$ which have outdegree two. The following technical lemma was proved in [31]. Again, although our definition is slightly different, the proof is identical.

Lemma 12 (Lemma 37 of [31]) Let $T, Z \in \mathbb{R}_{+}$, let $\mathcal{H}$ be a hierarchy for the rectangle $R$ which is good for the pair $(T, Z)$, and let $p>0$. There exists a rectangle $S \subset R$, called the 'pod' of $\mathcal{H}$, such that

$$
\operatorname{dim}(S) \leq \sum_{\text {seeds } u} \operatorname{dim}\left(R_{u}\right)
$$

and

$$
\sum_{\vec{N}_{G_{\mathcal{H}}}(v)=\{w\}} U\left(R_{w}, R_{v}\right) \geq U(S, R)-2 q M(\mathcal{H}) g(Z q) .
$$

We remark that although the rectangle $S$ is not necessarily unique, Lemma 12 allows us to select such a rectangle $S(\mathcal{H})$ for each good hierarchy $\mathcal{H} \in \mathcal{H}(R, T, Z)$. We shall refer to this rectangle as 'the pod of $\mathcal{H}$ '.

We shall use the following observation to bound $U(S, R)$ from below, and again later in the proof of Theorem 1.

Observation 13 There exists $C>0$ such that, for every $0<a<\infty$, we have

$$
\int_{0}^{a} g(z) d z \leq \frac{a}{2} \log \left(1+\frac{1}{a}\right)+C a .
$$

Proof Let $\varepsilon>0$ be such that Observation 4 holds when $z \leq \varepsilon$. Then, if $a \leq \varepsilon$ we have

$$
\int_{0}^{a} g(z) d z \leq \frac{1}{2} \int_{0}^{a}-\log z+2 z d z \leq \frac{a}{2} \log \frac{1}{a}+a+a^{2}
$$


as required. Moreover, since $g$ is decreasing, we have

$$
\int_{\varepsilon}^{a} g(z) d z \leq \operatorname{ag}(\varepsilon)
$$

and so the observation follows, since if $a>\varepsilon$ then $\int_{0}^{a} g(z) d z \leq 1+a g(\varepsilon)=O(a)$.

Finally, we shall use the following lemma, which follows from Lemma 16 of [31] (see also Lemma 7 of [29]).

Lemma 14 Let $q>0$ and $S \subset R$, with $\operatorname{dim}(S)=(a, b)$ and $\operatorname{dim}(R)=(A, B)$, where $A \leq B$. If $b \leq A$, then

$$
\frac{1}{q} U(S, R) \geq \frac{2}{q} \int_{0}^{A q} g(z) d z+(B-A) g(A q)-\frac{\phi(S)}{2} \log \left(1+\frac{1}{\phi(S) q}\right)-O(\phi(S)) .
$$

If $b>A$, then

$$
\frac{1}{q} U(S, R) \geq(A-a) g(b q)+(B-b) g(A q)
$$

Proof Assume first that $b \leq A$. By Lemma 16 of [31], the path integral is minimized by paths which follow the main diagonal as closely as possible. Assuming for simplicity that $a \leq b$, by following the piecewise linear path $(a q, b q) \rightarrow(b q, b q) \rightarrow$ $(A q, A q) \rightarrow(A q, B q)$ we obtain

$$
\frac{1}{q} U(S, R) \geq(b-a) g(b q)+\frac{2}{q} \int_{b q}^{A q} g(z) d z+(B-A) g(A q) .
$$

Now, by Observation 13, we have

$$
\frac{2}{q} \int_{0}^{b q} g(z) d z \leq b \log \left(1+\frac{1}{b q}\right)+O(b)
$$

and by Observation 4 we have $g(b q) \geq \frac{1}{2} \log (1+1 / b q)-O(1)$. (Note that inequality is trivial if $b q$ is not sufficiently small.) Hence

$$
(b-a) g(b q)-\frac{2}{q} \int_{0}^{b q} g(z) d z \geq-\frac{a+b}{2} \log \left(1+\frac{1}{b q}\right)-O(b),
$$

as required. The inequality for $b>A$ can be obtained by following the path $(a q, b q) \rightarrow(A q, b q) \rightarrow(A q, B q)$, and applying Lemma 16 of [31]. 


\section{The proof of Theorem 1}

In this section we shall put together the pieces and prove Theorem 1. Recall that, given $p>0$, we define $q=-\log (1-p) \sim p$ as $p \rightarrow 0$.

Proposition 15 Let $C>0$ and $\varepsilon>0$ be constants, let $p=p(C, \varepsilon)>0$ be sufficiently small, and let $R$ be a rectangle with dimensions $(a, b)$, where

$$
\frac{\varepsilon}{q} \leq a \leq b \leq \frac{C}{q} \log \left(\frac{1}{q}\right)
$$

Then

$\mathbb{P}_{p}([A \cap R]=R) \leq \exp \left(-\left[\frac{2}{q} \int_{0}^{a q} g(z) d z+(b-a) g(a q)\right]+\frac{O_{C}(1)}{\sqrt{q}}\left(\log \frac{1}{q}\right)^{3}\right)$

We remark that the constant implicit in the $O_{C}(1)$ term depends on the constant $C$, but not on the variables $p, a$ and $b$ (and also not on the constant $\varepsilon$ ).

We begin by defining some of the parameters we shall use. First, set $B=$ $C \log (1 / q)$, so that $a \leq b \leq B / q$, set $T=\sqrt{q}$, and set

$$
Z=\frac{1}{\sqrt{q}}\left(\log \frac{1}{q}\right)^{3}
$$

Let $S=S(\mathcal{H})$ denote the pod of a hierarchy $\mathcal{H}$, given by Lemma 12 .

Lemma 16 Let $C, \varepsilon, p>0, a, b \in \mathbb{N}$ and the rectangle $R$ be as in the statement of Proposition 15, and let $B, T$ and $Z$ be as defined above. Then

$$
\begin{aligned}
& \mathbb{P}_{p}(I(R)) \\
& \leq \sum_{\mathcal{H} \in \mathcal{H}(R, T, Z)} \exp \left[-\frac{1}{q} U(S, R)+O_{C}\left(N(\mathcal{H})\left(\log \frac{1}{q}\right)^{2}\right)\right] \prod_{\text {seeds } u} \mathbb{P}_{p}\left(I\left(R_{u}\right)\right) .
\end{aligned}
$$

Proof First note that by Observation 11 and Lemma 12, the pod $S=S(\mathcal{H}) \subset R$ of $\mathcal{H}$ satisfies

$$
\begin{aligned}
\sum_{\vec{N}_{G}\left(u_{i}\right)=\left\{v_{i}\right\}} s_{i} g\left(b_{i} q\right)+t_{i} g\left(a_{i} q\right) & \geq \frac{1}{q} \sum_{\vec{N}_{G \mathcal{H}}(u)=\{v\}} U\left(R_{v}, R_{u}\right) \\
& \geq \frac{1}{q} U(S, R)-2 M(\mathcal{H}) g(Z q),
\end{aligned}
$$

where $\left(a_{i}, b_{i}\right)$ and $\left(a_{i}+s_{i}, b_{i}+t_{i}\right)$ are the dimensions of $R_{v_{i}}$ and $R_{u_{i}}$ respectively. 
Now, by the definition of a hierarchy, we have $s_{i} \leq 2 T\left(a_{i}+s_{i}\right) \leq 3 T a_{i}$, and similarly $t_{i} \leq 3 T b_{i}$, for every pair $\left(u_{i}, v_{i}\right)$ with $N_{\vec{G}_{\mathcal{H}}}\left(u_{i}\right)=\left\{v_{i}\right\}$. Recall that $g(z)$ is decreasing, so

$$
\max \left\{g(Z q), g\left(a_{i} q\right), g\left(b_{i} q\right)\right\} \leq g(q) \leq \log \frac{1}{q},
$$

by Observation 4 (applied with $z=q$ ). Recall also that $a_{i}, b_{i} \leq b \leq B / q$.

By Observation 10, it follows that $s_{i} e^{2 g\left(a_{i} q\right)} \leq 4 B s_{i} / a_{i} q \leq 12 B \bar{T} / q$, and similarly $t_{i} e^{2 g\left(b_{i} q\right)} \leq 12 B T / q$. Thus

$$
g(Z q)+2 g\left(a_{i} q\right)+2 g\left(b_{i} q\right)+\left(q s_{i} t_{i}\right) e^{2 g\left(a_{i} q\right)+2 g\left(b_{i} q\right)} \leq 5 \log \frac{1}{q}+O\left(\frac{B^{2} T^{2}}{q}\right)
$$

and hence, since $T^{2}=q, B=O_{C}(\log (1 / q))$ and $M(\mathcal{H}) \leq N(\mathcal{H})$,

$$
\begin{aligned}
& 2 M(\mathcal{H}) g(Z q)+\sum_{\vec{N}_{G_{\mathcal{H}}}(u)=\{v\}}\left(2 g(b q)+2 g(a q)+(q s t) e^{2 g(b q)+2 g(a q)}\right) \\
& =O_{C}\left(N(\mathcal{H})\left(\log \frac{1}{q}\right)^{2}\right) .
\end{aligned}
$$

Hence, by Lemma 9, we have

$$
\prod_{\vec{N}_{G \mathcal{H}}(u)=\{v\}} \mathbb{P}_{p}\left(D\left(R_{v}, R_{u}\right)\right) \leq \exp \left[-\frac{1}{q} U(S, R)+O_{C}\left(N(\mathcal{H})\left(\log \frac{1}{q}\right)^{2}\right)\right],
$$

and so the lemma follows by Lemma 7.

We can now deduce Proposition 15 from Lemma 16. The main difficulty lies in the fact that there are too many hierarchies: there could be as many as $2^{1 / \sqrt{q}}$ vertices in $G_{\mathcal{H}}$, and for each vertex $u$ we have many choices for the rectangle $R_{u}$. However, most of these hierarchies have many seeds, and those with many large seeds have rather small weight in the sum. This turns out to be the key idea in the proof.

Indeed, given a hierarchy of $R$ which is good for $(T, Z)$, define a large seed to be one with $\phi\left(R_{u}\right) \geq Z / 3$. We make the following key observation.

Observation 17 Let $\mathcal{H} \in \mathcal{H}(R, T, Z)$, and assume that $4 T \leq 1$ and $Z \geq 6$. Then every vertex of $\mathcal{H}$ is either a seed, or lies above at least one large seed.

Proof By the definition of a good hierarchy, either $u$ is a seed, or $\phi\left(R_{u}\right)>Z$ and either $\vec{N}_{G_{\mathcal{H}}}(u)=\{v\}$ or $\vec{N}_{G_{\mathcal{H}}}(u)=\{v, w\}$. In the former case we have

$$
\phi\left(R_{v}\right) \geq(1-2 T) \phi\left(R_{u}\right) \geq \frac{Z}{2},
$$

since $4 T \leq 1$. In the latter case, we have $\phi\left(R_{v}\right)+\phi\left(R_{w}\right) \geq \phi\left(R_{u}\right)-2$, and so 


$$
\max \left\{\phi\left(R_{v}\right), \phi\left(R_{w}\right)\right\} \geq \frac{Z}{3},
$$

as required.

Let the number of large seeds in a hierarchy $\mathcal{H}$ be denoted $m(\mathcal{H})$.

Proof of Proposition 15. Let $R \subset[n]^{2}, p>0, B=C \log (1 / q), T=\sqrt{q}$, and $Z=(1 / \sqrt{q})\left(\log \frac{1}{q}\right)^{3}$ be as described above, and suppose that $\mathcal{H}$ is a hierarchy for $R$ which is good for the pair $(T, Z)$. Recall that $p>0$ is chosen sufficiently small, and that $a \leq b \leq B / q$. By Lemma $5, \mathcal{H}$ has height at most $(10 / \sqrt{q}) \log (1 / q)$, and hence the number of vertices $N(\mathcal{H})$ in $G_{\mathcal{H}}$ satisfies

$$
N(\mathcal{H}) \leq 2 m(\mathcal{H}) \cdot h(\mathcal{H})=O\left(\frac{m(\mathcal{H})}{\sqrt{q}} \log \frac{1}{q}\right)
$$

Therefore, the number of hierarchies with $m$ large seeds (i.e., with $m(\mathcal{H})=m$ ) is at most

$$
\sum_{N}\left(\frac{B}{q}\right)^{4 N} \leq \exp \left(O(1) \frac{m}{\sqrt{q}}\left(\log \frac{1}{q}\right)^{2}\right)
$$

Now, for each hierarchy $\mathcal{H}$, define

$$
X(\mathcal{H}):=\sum_{\text {seeds } u} \phi\left(R_{u}\right)
$$

and note that $X(\mathcal{H}) \geq \frac{m(\mathcal{H}) Z}{3}$, and that $\phi(S(\mathcal{H})) \leq X(\mathcal{H})$, by Lemma 12. By Lemma 2, for every seed $R_{u}$ we have

$$
\mathbb{P}_{p}\left(I\left(R_{u}\right)\right) \leq 3^{\phi\left(R_{u}\right)} \exp \left(-\phi\left(R_{u}\right) g(Z q)\right),
$$

since $\operatorname{sh}\left(R_{u}\right) \leq Z=o(1 / q)$ as $q \rightarrow 0$, and $g(z)$ is decreasing in $z$. Thus

$$
\prod_{\text {seeds } u} \mathbb{P}_{p}\left(I\left(R_{u}\right)\right) \leq 3^{X(\mathcal{H})} \exp (-X(\mathcal{H}) g(Z q))
$$

We split into two cases. The first is easier to handle, and we shall not have to approximate too carefully; in the second the calculation is much tighter.

Case 1: $\lg (S)>a$.

We have, by Lemma 16 combined with (2) and (4),

$$
\begin{aligned}
\mathbb{P}_{p}(I(R)) \leq & \sum_{\mathcal{H} \in \mathcal{H}(R, T, Z)} 3^{X(\mathcal{H})} \exp \left[-\frac{1}{q} U(S, R)-X(\mathcal{H}) g(Z q)\right. \\
& \left.+O_{C}\left(\frac{m(\mathcal{H})}{\sqrt{q}}\left(\log \frac{1}{q}\right)^{3}\right)\right] .
\end{aligned}
$$


Recall that $a<\phi(S) \leq X(\mathcal{H})$, by Lemma 12 , and so $\frac{1}{q} U(S, R) \geq(b-X(\mathcal{H})) g(a q)$, by Lemma 14 . Hence the summand above is at most

$$
3^{X(\mathcal{H})} \exp \left[-b g(a q)-X(\mathcal{H})(g(Z q)-g(a q))+O_{C}\left(\frac{m(\mathcal{H})}{\sqrt{q}}\left(\log \frac{1}{q}\right)^{3}\right)\right]
$$

Now, since $g$ is decreasing, $X(\mathcal{H}) \geq m(\mathcal{H}) Z / 3$ and $a / Z \geq q^{-1 / 3}$, by Observation 4 we have

$X(\mathcal{H})(g(Z q)-g(a q)) \geq \frac{X(\mathcal{H})}{7} \log \left(\frac{1}{q}\right)=\frac{1}{o(1)} m(\mathcal{H}) Z=\frac{1}{o(1)} \frac{m(\mathcal{H})}{\sqrt{q}}\left(\log \frac{1}{q}\right)^{3}$

as $q \rightarrow 0$. It follows that

$$
\begin{aligned}
\mathbb{P}_{p}(I(R)) & \leq \sum_{\mathcal{H}} \exp \left(-b g(a q)-\frac{X(\mathcal{H})}{8} \log \frac{1}{q}\right) \\
& \leq \exp \left(-\frac{2}{q} \int_{0}^{a q} g(z) d z-b g(a q)\right),
\end{aligned}
$$

as required. Indeed, we showed that $X(\mathcal{H})(g(Z q)-g(a q))$ is at least $\frac{X(\mathcal{H})}{7} \log \frac{1}{q}$, and much bigger than $\frac{m(\mathcal{H})}{\sqrt{q}}\left(\log \frac{1}{q}\right)^{3}$, so the first inequality holds. For the last inequality, first note that

$$
\frac{2}{q} \int_{0}^{a q} g(z) d z \leq a \log \left(1+\frac{1}{a q}\right)+O(a)=o\left(X(\mathcal{H}) \log \frac{1}{q}\right)
$$

as $q \rightarrow 0$, by Observation 13. Here we used the facts that $a q \geq \varepsilon$ and $a<X(\mathcal{H})$. Finally, note that, by (3), there are at most $e^{X}$ hierarchies with $X(\mathcal{H})=X$. Hence we obtain a geometrically decreasing sum over $X$, and the claimed bound follows.

Case 2: $\lg (S) \leq a$.

By Lemma 14, and since $\phi(S) \leq X(\mathcal{H})$, we have

$$
\begin{aligned}
\frac{1}{q} U(S, R) \geq & \frac{2}{q} \int_{0}^{a q} g(z) d z+(b-a) g(a q) \\
& -\frac{X(\mathcal{H})}{2} \log \left(1+\frac{1}{X(\mathcal{H}) q}\right)-O(X(\mathcal{H})) .
\end{aligned}
$$


Hence, by (2), (4) and Lemma 16, we have

$$
\begin{aligned}
\mathbb{P}_{p}(I(R)) \leq & \sum_{\mathcal{H} \in \mathcal{H}(R, T, Z)} \exp \left[-\frac{2}{q} \int_{0}^{a q} g(z) d z-(b-a) g(a q)\right. \\
& +\frac{X(\mathcal{H})}{2} \log \left(1+\frac{1}{X(\mathcal{H}) q}\right)+O(X(\mathcal{H})) \\
& \left.+O_{C}\left(\frac{m(\mathcal{H})}{\sqrt{q}}\left(\log \frac{1}{q}\right)^{3}\right)-X(\mathcal{H}) g(Z q)\right] .
\end{aligned}
$$

But by Observation 4,

$$
X(\mathcal{H})\left(\frac{1}{2} \log \left(1+\frac{1}{X(\mathcal{H}) q}\right)+c_{1}-g(Z q)\right) \leq-\frac{X(\mathcal{H})}{2} \log \left(\frac{X(\mathcal{H})}{C_{1} Z(1+X(\mathcal{H}) q)}\right)
$$

where $C_{1}=e^{2 c_{1}+1}$. Note that, for any $u, v>0$, the function $x \log \left(\frac{x}{u x+v}\right)$ is increasing if $x \geq u x+v$, and recall that $X(\mathcal{H}) \geq \frac{m(\mathcal{H}) Z}{3}$. Thus, the right-hand side above is decreasing in $X(\mathcal{H})$ if $m(\mathcal{H})$ is sufficiently large, and hence either $m(\mathcal{H})=O(1)$, or

$$
-\frac{X(\mathcal{H})}{2} \log \left(\frac{X(\mathcal{H})}{C_{1} Z(1+X(\mathcal{H}) q)}\right) \leq-\frac{m(\mathcal{H}) Z}{6} \log \left(\frac{m(\mathcal{H})}{4 C_{1}}\right)
$$

and $m(\mathcal{H}) Z q \leq 1$, or the left-hand side is at most $-\frac{m(\mathcal{H}) Z}{6} \log (1 / q)$.

Finally, recalling that $Z=\frac{1}{\sqrt{q}} \log ^{3}(1 / q)$, we have

$$
\begin{aligned}
& -\frac{m(\mathcal{H}) Z}{6} \log \left(\frac{m(\mathcal{H})}{4 C_{1}}\right)+O_{C}\left(\frac{m(\mathcal{H})}{\sqrt{q}}\left(\log \frac{1}{q}\right)^{3}\right) \\
& \leq \frac{O_{C}(1)}{\sqrt{q}}\left(\log \frac{1}{q}\right)^{3}-\frac{m(\mathcal{H})}{\sqrt{q}}\left(\log \frac{1}{q}\right)^{3}
\end{aligned}
$$

since either $m(\mathcal{H})=O_{C}(1)$, or the first (negative) term dominates.

Putting these various bounds together gives

$$
\begin{aligned}
\mathbb{P}_{p}(I(R)) \leq & \sum_{\mathcal{H} \in \mathcal{H}(R, T, Z)} \exp \left[-\frac{2}{q} \int_{0}^{a q} g(z) d z-(b-a) g(a q)\right. \\
& \left.+\frac{O_{C}(1)-m(\mathcal{H})}{\sqrt{q}}\left(\log \frac{1}{q}\right)^{3}\right] .
\end{aligned}
$$


Hence, using (3), and summing over $m(\mathcal{H})$, we obtain

$$
\mathbb{P}_{p}(I(R)) \leq \exp \left[-\frac{2}{q} \int_{0}^{a q} g(z) d z-(b-a) g(a q)+\frac{O_{C}(1)}{\sqrt{q}}\left(\log \frac{1}{q}\right)^{3}\right]
$$

as required.

Before deducing Theorem 1 from Proposition 15, we need to recall the following fact from [31], and to make an easy observation.

Lemma 18 (Proposition 5 of [31])

$$
\int_{0}^{\infty} g(z) d z=\frac{\pi^{2}}{18}
$$

The following observation follows almost immediately from Lemma 18.

Observation 19 Let $p>0$ be sufficiently small, and let $a, b \in \mathbb{R}_{+}$, with $a \leq b$ and $b \geq B / 2 p$, where $B=10 \log (1 / p)$. Then

$$
\frac{2}{q} \int_{0}^{a q} g(z) d z+(b-a) g(a q) \geq \frac{2 \lambda}{q}-1
$$

where $\lambda=\pi^{2} / 18$.

Proof If $a \leq B / 4 p$, then this follows since $\int_{a q}^{\infty} g(z) d z=O(g(a q))$, uniformly over $a \in(0, \infty)$, and so

$$
(b-a) g(a q)-\frac{2}{q} \int_{a q}^{\infty} g(z) d z \geq\left(\frac{B}{4 p}\right) g(a q)-O\left(\frac{g(a q)}{q}\right)>0 .
$$

If $a \geq B / 4 p$ then it holds because $g(z) \leq 2 e^{-z}$ for $z$ large, and so

$$
\frac{2}{q} \int_{a q}^{\infty} g(z) d z \leq \frac{4}{q} e^{-a q} \leq \frac{4}{q} e^{-B / 5} \leq 1
$$

as required.

Finally, we deduce Theorem 1 from Proposition 15.

Proof of Theorem 1. Let $C_{2}>0$ be a large constant to be chosen later, let $n \in \mathbb{N}$ be sufficiently large, and let

$$
p=\frac{\pi^{2}}{18 \log n}-\frac{C_{2}(\log \log n)^{3}}{(\log n)^{3 / 2}} .
$$


Note that $q=-\log (1-p)<p+p^{2}$, and so $q$ also satisfies this equality (with a slightly different constant $C_{2}$ ).

Let the elements of $A \subset[n]^{2}$ be chosen independently at random, each with probability $p$, and suppose that $A$ percolates. Then, by Lemma 8 , there exists a rectangle $R \subset[n]^{2}$, which is internally spanned by $A$, and with $B / 2 p \leq \lg (R) \leq B / p$, where $B=10 \log (1 / p)$. Let $\operatorname{dim}(R)=(a, b)$, and assume without loss of generality that $a \leq b$. There are at most $n^{2}(B / p)^{2}$ potential such rectangles, and each is internally spanned with probability at most

$\mathbb{P}_{p}([A \cap R]=R) \leq \exp \left(-\left[\frac{2}{q} \int_{0}^{a q} g(z) d z+(b-a) g(a q)\right]+\frac{O(1)}{\sqrt{q}}\left(\log \frac{1}{q}\right)^{3}\right)$

if $\operatorname{sh}(R) \geq 1 / q$, by Proposition 15 , and with probability at most

$$
e^{-b g(a q)} \leq e^{-B / 40 p}=p^{1 / 4 p} \leq\left(\frac{1}{n}\right)^{100}
$$

if $a=\operatorname{sh}(R) \leq 1 / q$ and $n$ is sufficiently large, since $g(a q) \geq g(1)=-\log \beta\left(\frac{e-1}{e}\right)>$ $1 / 20$ and $b \geq B / 2 p$. Note that, since we apply Proposition 15 with $C=10$, we obtain an absolute constant $O(1)$ in the expression above.

By Observation 19, we have

$$
\frac{2}{q} \int_{0}^{a q} g(z) d z+(b-a) g(a q) \geq \frac{2 \lambda}{q}-1
$$

where $\lambda=\pi^{2} / 18$. Thus, using the identity $\frac{1}{x-y}=\frac{1}{x}+\frac{y}{x(x-y)}$, this gives, as $n \rightarrow \infty$,

$$
\begin{aligned}
\mathbb{P}_{p}\left([A]=[n]^{2}\right) & \leq n^{2}(B / p)^{2} \exp \left(-\frac{2 \lambda}{q}+\frac{O(1)}{\sqrt{q}}\left(\log \frac{1}{q}\right)^{3}\right) \\
& \leq n^{2}(B / p)^{2} \exp \left(-2 \log n-\frac{C_{2}}{\lambda}(\log \log n)^{3} \sqrt{\log n}+\frac{O(1)}{\sqrt{q}}\left(\log \frac{1}{q}\right)^{3}\right) \\
& \leq n^{2}(\log n)^{3} \exp \left(-2 \log n-(\log \log n)^{3} \sqrt{\log n}\right) \rightarrow 0
\end{aligned}
$$

if $C_{2}$ is sufficiently large, as required.

\section{Extensions and open questions}

In this paper we have studied bootstrap percolation on one particular graph, the twodimensional grid with nearest-neighbour bonds. It is natural to ask whether our method can be applied to bootstrap percolation on other graphs; here we shall discuss two such possible generalizations. 
The most obvious (and most extensively studied) generalization is to consider bootstrap percolation in $d$ dimensions (i.e., on the graph $[n]^{d}$ ), with nearest neighbour interaction and threshold $2 \leq r \leq d$ (as studied in, for example, $[2,6,8,16,17,40]$ ). The sharp metastability thresholds for these models (with $d$ fixed, and as $n \rightarrow \infty$ ) were determined in [8], and it is likely that the methods of this paper (and those of [28]) could be adapted to give improved bounds in the case of $r=2$ and general $d$.

Problem 1 Bound the second term in the asymptotic expansion of $p_{c}\left([n]^{d}, 2\right)$ as $n \rightarrow \infty$.

The case $r \geq 3$ is more complicated, and the following problem is likely to be difficult.

Problem 2 Bound the second term in the asymptotic expansion of $p_{c}\left([n]^{3}, 3\right)$ as $n \rightarrow \infty$.

We remark that in very high dimensions $(d \gg \log n$, say) new ideas are required, and much less is known in general. However, results analogous to Theorem 1 have been proved in the special cases $r=2$ and $r=d$, see [5,7].

A second natural generalization is to consider bootstrap percolation in two dimensions, but with a different update rule. For example, in the 'modified' bootstrap process (see [32]), a vertex is infected if at least one of its neighbours in each dimension is already infected; in the ' $k$-cross' process (see $[33,15])$, a vertex $v$ is infected if at least $k$ vertices in the cross-shaped set

$$
\bigcup_{0 \neq j \in[-k+1, k-1]}\{v+(0, j), v+(j, 0)\}
$$

are previously infected; and in the Froböse process (introduced by Froböse [25] in 1989) a site of $[n]^{2}$ is infected if it has one already-infected neighbour in each dimension, along with the next-nearest neighbour in the corner between them. In general, one could consider an arbitrary neighbourhood $N(v)$ of each vertex $v$, an arbitrary (monotone) family $\mathcal{A}(v)$ of subsets of $N(v)$, and say that $v$ becomes infected if the already-infected subset of its neighbours is in $\mathcal{A}(v)$.

Holroyd [31] (see also [32]) determined the sharp threshold for the modified and Froböse models, and Holroyd, Liggett and Romik [33] did so for the $k$-cross process for all fixed $k \in \mathbb{N}$. Moreover, Duminil-Copin and Holroyd [22] have recently shown, for a large family of such models (including all of the examples above, and other similar models), that there exists a sharp metastability threshold. It is not unreasonable to hope that our method (together with that of [28]) might yield improved bounds on the critical probability for a more general collection of bootstrap processes, of the type considered in [22]. Indeed, for two of the processes described above this is the case.

Let $p_{c}^{(\mathrm{F})}\left([n]^{2}\right)$ denote the critical probability for percolation in the Froböse process on $[n]^{2}$, and let $p_{c}^{(+)}\left([n]^{2}, k\right)$ denote the critical probability for percolation in the $k$-cross process. The upper bounds in the following theorem were proved by Gravner and Holroyd [28] (for the Froböse model) and by Bringmann and Mahlburg [15] (for the $k$-cross process). The lower bounds follow by the methods of this paper. 


\section{Theorem 20}

$$
p_{c}^{(\mathrm{F})}\left([n]^{2}\right)=\frac{\pi^{2}}{6 \log n}-\frac{1}{(\log n)^{3 / 2+o(1)}} .
$$

as $n \rightarrow \infty$. Let $k \in \mathbb{N}$, and let $\lambda_{k}=\pi^{2} / 3 k(k+1)$. Then

$$
p_{c}^{(+)}\left([n]^{2}, k\right)=\frac{\lambda_{k}}{\log n}-\frac{1}{(\log n)^{3 / 2+o(1)}}
$$

as $n \rightarrow \infty$.

In fact the bounds we prove (and those from $[15,28]$ ) are a little stronger than those stated above; they are like the bounds in Theorem 1.

Sketch of proof of Theorem 20. For the first part, it suffices to show that (in the Froböse process) on $R=[m] \times[n]$, all spanning sets have size at least $m+n-1$. The result then follows in exactly the same way as Theorem 1 . Indeed, simply replace the function $g$ by the function

$$
h(z)=-\log \left(1-e^{-z}\right)
$$

and note that a rectangle is crossed if, and only if, it has no empty column. The rest of the proof carries over essentially verbatim, the key point being that $\int_{0}^{x} h(z) d z \sim$ $x \log (1 / x)$ when $x \rightarrow 0$, and $\mathbb{P}_{p}\left(I\left(R_{u}\right)\right) \approx \exp \left(-\phi\left(R_{u}\right) h(a q)\right)$ when $u$ is a seed, so the corresponding terms in the final calculation are of the same order.

We shall give two proofs that if $[A]=R$ then $|A| \geq m+n-1$. The first is standard, using Proposition 30 of [31] and induction on $\phi(R)$ (see Lemma 7 of [4] or Problem 35 of [14]). For the second, consider the (bipartite) graph $G$ whose vertices are the rows and columns of $R$, with an edge from row $x$ to column $y$ if and only if $(x, y) \in A$.

To prove that $G$ has at least $m+n-1$ edges, we shall show that it is connected. Indeed, if $G$ is not connected then exists a set of rows $X$ and a set of columns $Y$ such that $A \subset S=(X \cap Y) \cup\left(X^{c} \cap Y^{c}\right)$. But then $[S]=S \neq R$, so $A$ does not percolate, as required.

For the second part, we need the following idea from [33]: first couple the $k$-cross process with an 'enhanced process' (see [33, Section 5]) in which the closed sets are rectangles. In the enhanced process the minimum number of sites required to infect an $[m] \times[n]$ rectangle is about $(m+n) / k$, which is also the typical number required. (To prove this, apply the standard proof, by induction on $m+n$.)

The result now follows by the proof of Theorem 1, replacing the function $g$ by the function $-\log f\left(e^{-z}\right)$, where $f:[0,1] \rightarrow[0,1]$ is decreasing and satisfies

$$
f^{k}-f^{k+1}=x^{k}-x^{k+1}
$$

and noting that if a rectangle is crossed in the enhanced process, then it has no ' $k$-gap' of $k$ successive empty columns (see Lemma 12 of [33]). 
We obtain a sufficiently strong bound on $\mathbb{P}_{p}\left(I\left(R_{u}\right)\right)$, where $u$ is a seed, using the proof of Lemma 2; this works because our lower bound on $|A \cap R|$ is also the typical size of a percolating set in $R$ when $\phi(R) \ll 1 / p$. It follows that the contribution of the large seeds to the final calculation is of the same order as that of the integral $\int_{0}^{x}-\log f\left(e^{-z}\right) d z$, where $x$ is the semi-perimeter of the pod. Modulo a little basic analysis, the rest of the proof works as above; we leave the details to the reader.

Gravner and Holroyd [28] also improved the upper bounds for the modified process. However, the proof of Theorem 1 does not work for the modified process, since we do not have a result analogous to Lemma 2. In particular, it is possible to internally span an $m \times n$ rectangle with $\max \{m, n\}$ infected sites, but the proportion of such minimal-size sets which percolate is very small.

Let $p_{c}^{(\mathrm{M})}\left([n]^{d}\right)$ denote the critical probability for percolation in the modified bootstrap process on the graph $[n]^{d}$, i.e., the infimum over $p$ such that the probability of percolation is at least $1 / 2$. We have the following conjecture; it is the analogue of Conjecture 1 for the modified process.

Conjecture 2 As $n \rightarrow \infty$,

$$
p_{c}^{(\mathrm{M})}\left([n]^{2}\right)=\frac{\pi^{2}}{6 \log n}-\frac{1}{(\log n)^{3 / 2+o(1)}} .
$$

Given a rectangle $R$, we say that a set $A \subset R$ is a minimal percolating set if $A$ spans $R$, but no proper subset of $A$ does so (see [37], for example). Given $m \geq n$ and $x \geq 0$, let $F(m, n, x)$ denote the number of minimal percolating sets of size $m+x$ in modified bootstrap percolation on $R=[m] \times[n]$. We remark that Conjecture 2 would follow from the method of this paper, together with following bound:

$$
F(m, n, x) \leq n^{m-n+2 x+o(n)} .
$$

Note that even if we restrict ourselves to 'threshold' models, in which a vertex is infected if at least $r$ elements of its neighbourhood are infected, we still run into similar problems. Indeed, consider the model in which a vertex is infected if at least four of its eight neighbours (including diagonals) are infected. A typical seed $R$ is shaped like an octagon, and the number of infected sites used to fill $R$ (in the random process) is roughly $\phi(R)$ (which we define to be the number of external vertices plus the number of external edges), while the minimal number required to span $R$ is only $\phi(R) / 2$.

Finally, returning to the standard bootstrap process, recall that Theorem 1 determines the second term of $p_{c}\left([n]^{2}, 2\right)$ up to a poly $(\log \log n)$-factor. We ask whether this error term can be removed.

Problem 3 Determine $\alpha \in[0,3]$, if it exists, such that

$$
p_{c}\left([n]^{2}, 2\right)=\frac{\pi^{2}}{18 \log n}-\frac{(\log \log n)^{\alpha+o(1)}}{(\log n)^{3 / 2}}
$$

as $n \rightarrow \infty$. 
As usual in bootstrap percolation, it would not be unreasonable to suspect that the upper bound in Theorem 1 is closer to the truth.

Open Access This article is distributed under the terms of the Creative Commons Attribution Noncommercial License which permits any noncommercial use, distribution, and reproduction in any medium, provided the original author(s) and source are credited.

\section{References}

1. Adler, J., Lev, U.: Bootstrap percolation: visualizations and applications. Braz. J. Phys. 33, 641-644 (2003)

2. Aizenman, M., Lebowitz, J.L.: Metastability effects in bootstrap percolation. J. Phys. A. 21, 3801-3813 (1988)

3. Balogh, J.: Graph parameters and bootstrap percolation. Ph.D. Dissertation, Memphis (2001)

4. Balogh, J., Bollobás, B.: Bootstrap percolation on the hypercube. Prob. Theory Relat. Fields 134, 624648 (2006)

5. Balogh, J., Bollobás, B., Morris, R.: Majority bootstrap percolation on the hypercube. Combin. Probab. Comput. 18, 17-51 (2009)

6. Balogh, J., Bollobás, B., Morris, R.: Bootstrap percolation in three dimensions. Ann. Probab. 37, 13291380 (2009)

7. Balogh, J., Bollobás, B., Morris, R.: Bootstrap percolation in high dimensions. Combin. Probab. Comput. arXiv:0907.3097 (2010, to appear)

8. Balogh, J., Bollobás, B., Duminil-Copin, H., Morris, R.: The sharp threshold for bootstrap percolation in all dimensions. arXiv:1010.3326 (2010, submitted)

9. Balogh, J., Peres, Y., Pete, G.: Bootstrap percolation on infinite trees and non-amenable groups. Combin. Probab. Comput. 15, 715-730 (2006)

10. Balogh, J., Pittel, B.: Bootstrap percolation on random regular graphs. Random Struct. Algorithms 30, 257-286 (2007)

11. Baxter, G.J., Dorogovtsev, S.N., Goltsev, A.V., Mendes, J.F.F.: Bootstrap percolation on complex networks. Phys. Rev. E 82. arXiv:1003.5583 (2010, to appear)

12. van den Berg, J., Kesten, H.: Inequalities with applications to percolation and reliability. J. Appl. Probab. 22, 556-589 (1985)

13. Biskup, M., Schonmann, R.H.: Metastable behavior for bootstrap percolation on regular trees. J. Stat. Phys. 136(4), 667-676 (2009)

14. Bollobás, B.: The Art of Mathematics: Coffee Time in Memphis. Cambridge University Press, Cambridge (2006)

15. Bringmann, K., Mahlburg, K.: Improved bounds on metastability thresholds and probabilities for generalized bootstrap percolation. arXiv:1001.1977 (2010)

16. Cerf, R., Cirillo, E.N.M.: Finite size scaling in three-dimensional bootstrap percolation. Ann. Probab. 27, 1837-1850 (1999)

17. Cerf, R., Manzo, F.: The threshold regime of finite volume bootstrap percolation. Stoch. Proc. Appl. 101, 69-82 (2002)

18. Cerf, R., Manzo, F.: A $d$-dimensional nucleation and growth model. arXiv:1001.3990 (2010)

19. Chalupa, J., Leath, P.L., Reich, G.R.: Bootstrap percolation on a Bethe latice. J. Phys. C. 12, L31-L35 (1979)

20. Dehghanpour, P., Schonmann, R.H.: Metropolis dynamics relaxation via nucleation and growth. Commun. Math. Phys. 188, 89-119 (1997)

21. Dehghanpour, P., Schonmann, R.H.: A nucleation-and-growth model. Probab. Theory Relat. Fields 107, 123-135 (1997)

22. Duminil-Copin, H., Holroyd, A.: Sharp metastability for threshold growth models. (2010, in preparation)

23. Fontes, L.R., Schonmann, R.H.: Bootstrap percolation on homogeneous trees has 2 phase transitions. J. Stat. Phys. 132, 839-861 (2008)

24. Fontes, L.R., Schonmann, R.H., Sidoravicius, V.: Stretched exponential fixation in stochastic Ising models at zero temperature. Commun. Math. Phys. 228, 495-518 (2002) 
25. Froböse, K.: Finite-size effects in a cellular automaton for diffusion. J. Stat. Phys. 55(5-6), 12851292 (1989)

26. Granovetter, M.: Threshold models of collective behavior. Am. J. Sociol. 83, 1420-1443 (1978)

27. Gravner, J., Griffeath, D.: Threshold growth dynamics. Trans. Am. Math. Soc. 340, 837-870 (1993)

28. Gravner, J., Holroyd, A.E.: Slow convergence in bootstrap percolation. Ann. Appl. Probab. 18, 909$928(2008)$

29. Gravner, J., Holroyd, A.E.: Local bootstrap percolation. Electron. J. Probab. 14, 385-399 (2009)

30. De Gregorio, P., Lawlor, A., Bradley, P., Dawson, K.A.: Exact solution of a jamming transition: closed equations for a bootstrap percolation problem. Proc. Natl. Acad. Sci. USA 102(16), 5669-5673 (2005)

31. Holroyd, A.: Sharp metastability threshold for two-dimensional bootstrap percolation. Prob. Theory Relat. Fields 125, 195-224 (2003)

32. Holroyd, A.: The metastability threshold for modified bootstrap percolation in $d$ dimensions. Electron. J. Probab. 11, 418-433 (2006)

33. Holroyd, A.E., Liggett, T.M., Romik, D.: Integrals, partitions, and cellular automata. Trans. Am. Math. Soc. 356(8), 3349-3368 (2004)

34. Janson, S.: On percolation in Random Graphs with given vertex degrees. Electron. J. Probab. 14, 86-118 (2009)

35. Janson, S., Łuczak, T., Turova, T., Vallier, T.: Bootstrap percolation on the random graph $G_{n, p}$. arXiv: 1012.3535

36. Kesten, H., Schonmann, R.H.: On some growth models with a small parameter. Probab. Theory Relat. Fields 101, 435-468 (1995)

37. Morris, R.: Minmal percolating sets in bootstrap percolation. Electron. J. Combin. 16, Research Paper 2, $20 \mathrm{pp}(2009)$

38. Morris, R.: Zero-temperature Glauber dynamics on $\mathbb{Z}^{d}$. Probab. Theory Relat. Fields. arXiv:0809.0353 (2010, to appear)

39. Richardson, D.: Random growth in a tessellation. Proc. Cambridge Philos. Soc. 74, 515-528 (1973)

40. Schonmann, R.H.: On the behaviour of some cellular automata related to bootstrap percolation. Ann. Probab. 20, 174-193 (1992)

41. Watts, D.J.: A simple model of global cascades on random networks. Proc. Natl. Acad. Sci. 99, 5766$5771(2002)$

42. Winkler, P.: Mathematical Puzzles: A Connoisseur's Collection. A K Peters Ltd., Natick (2004) 